\title{
COVID-19 Vaccine: Side Effects After the First Dose of the Oxford AstraZeneca Vaccine Among Health Professionals in Low-Income Country: Ethiopia
}

\author{
Yoseph Solomon (iD) ${ }^{1, *}$ \\ Tewodros Eshete $\mathbb{D}^{2, *}$ \\ Bersabeh Mekashal,* \\ Wubshet Assefa ${ }^{3, *}$ \\ 'Department of Surgery, School of \\ Medicine, Debre Markos University, \\ Debre Markos, Ethiopia; ${ }^{2}$ Department of \\ Health Informatics, College of Health \\ Sciences, Debre Markos University, \\ Debre Markos, Ethiopia; ${ }^{3}$ Department of \\ Pathology, School of Medicine, Debre \\ Markos University, Debre Markos, \\ Ethiopia
}

*These authors contributed equally to this work
Correspondence: Tewodros Eshete Department of Health Informatics, College of Health Sciences, Debre Markos University, P.O. Box: 269, Debre Markos, Ethiopia

Tel +25 I 933086482

Email teddyeshet143@gmail.com
Purpose: The Oxford-AstraZeneca is one of COVID-19 vaccine which is expected to be mass-produced and plays a critical role in controlling the pandemic that the globe faced. Ethiopia launched the AstraZeneca vaccination and planned to vaccinate $20 \%$ of the population by the end of 2021. Health care professionals are one of the eligible groups of the community to receive the vaccine with priority. Although individuals are advised to take the vaccine to protect themselves and the people around them from COVID-19 infection, many are doubtful about the consequences of the vaccine. So, this study assessed the immediate symptoms associated with taking the Oxford-AstraZeneca COVID-19 vaccine.

Methods: This online study was conducted from April 15 to 30, 2021 at a national level across health care providers who took their first dose of Oxford-AstraZeneca vaccine in Ethiopia.

Results: There were 672 study participants engaged in this study and around $75.8 \%$ of health care providers who took the vaccine had injection site symptoms like pain $(65.48 \%)$ and tenderness (57.89\%). Most of them (60\%) developed their injection site symptom within 12 hours after vaccination and the symptoms lasted for about 24-72 hours on most $(63.53 \%)$ of the participants. Mild symptoms were identified among $70.98 \%$ of the study participants; tiredness and headache were the most reported symptoms with $52.08 \%$ and $50.15 \%$, respectively. Only $6.1 \%$ of participants reported severe symptoms.

Conclusion: As like that of other vaccines, the Oxford-AstraZeneca COVID-19 vaccine has some adverse effects and most side effects peaked within the first 24 hours following vaccination and usually lasted 1-3 days. Severe symptoms were uncommon, but they were found to be a major reason why vaccine recipients did not recommend it to others and did not plan to take their second dose. After receiving the COVID-19 vaccination, recipients should be advised about potential vaccine symptoms, how to handle them, and when and where to seek additional guidance if necessary.

Keywords: COVID-19, Ethiopia, health care professionals, Oxford-AstraZeneca

\section{Introduction}

Globally, as of 28 June 2021, a total of 180,817,269 confirmed cases of COVID-19, including 3,923,238 deaths were reported to World Health Organization (WHO). On a similar date of access, there have been 275,935 confirmed cases of COVID-19 and 4314 deaths reported in Ethiopia. ${ }^{1}$ Besides this, the pandemic has brought the worst global economic contraction of the past 80 years through its multi-sectoral impact such as unemployment, poverty, human development, investment, trade, tourism, transport, manufacturing industries, commodity prices, small and medium-sized 
enterprises. $^{2}$ To overcome the ongoing challenges, the WHO has created Strategic Preparedness and Response Plan 2021 (SPRP2021) which translates the previously learned knowledge into coordinated action. The plan also focuses on strengthening researches and innovation for the effective delivery of safe vaccines as part of the overall strategy to successfully confront the COVID-19 pandemic. $^{3}$

To date, the scientific community is working collaboratively and aggressively to develop an efficient and effective vaccine against the SARS-CoV-2 virus with minimal side effects and complications. ${ }^{4}$ Across the globe, there are three major categories of COVID-19 vaccines until the end of June 2021:

1. Messenger ribonucleic acid (mRNA) vaccines: BNT162b2 (Pfizer-BioNTech, New York, New York) and mRNA-1273 (Moderna, Inc., Cambridge, Massachusetts),

2. Adenoviral vector vaccines: ChAdOx1 nCoV-19 (AstraZeneca-Oxford), Gam-COVID-Vac (Gamaleya National Centre of Epidemiology and Microbiology), Ad26.COV2.S (Johnson \& Johnson), and Ad5-nCoV (CanSinoBIO), and

3. Inactivated whole-virus vaccines: BBIBP-CorV (Sinofarm) and CoronaVac (Sinovac Life Sciences). ${ }^{5}$

Unlike many other vaccines, which are costly and must be stored at extremely low temperatures, the OxfordAstraZeneca vaccine can be stored in a regular refrigerator and costs only a few dollars per dose. Furthermore, it is expected to be mass-produced and may play an important role in curbing the pandemic. ${ }^{6}$

It has been few months since Ethiopia launched the Oxford AstraZeneca vaccine for COVID-19 and aimed to vaccinate $20 \%$ of the population by the end of 2021 . As per the National Deployment and Vaccination Plan (NDVP) of Ethiopia, developed following the WHO Prioritizing Roadmap, the first candidates to receive this vaccine are population groups with relatively higher risk of contracting the virus. Given the day-to-day interactions with myriads of patients, health care professionals are one of the eligible groups of the community to receive the vaccine with priority. ${ }^{7}$

Vaccine uptake in low- and middle-income countries is generally explained by a desire for personal protection against COVID-19, despite the most common reason for hesitancy being adverse side effects after vaccination. ${ }^{8-10}$ Decisions about health care, including whether or not to participate in COVID-19 vaccination, are based on weighing the potential costs of participation against the expected benefits. As a result, increasing vaccination uptake requires addressing concerns by delivering evidence-based information as part of bigger awareness campaigns and individual conversations. ${ }^{11}$ Although individuals are advised to take the vaccine to protect themselves and the people around them from COVID-19 infection, many including health professionals are doubtful about the possible side effects, consequences, and effectiveness of the vaccine. ${ }^{12,13}$

Manufacturers of vaccines include a list of potential post-vaccination side effects with their products. The occurrence of adverse vaccine reactions shows that the vaccine is effective and that immunity to the disease is increasing. The following is a list of these reactions, according to the Centers for Disease Control and Prevention (CDC) and other studies: Symptoms at the injection site (swelling, pain, and redness) as well as systemic effects (back pain, tiredness, headache, muscle pain, joint pain, chills, fever, and nausea). ${ }^{14-17}$

Countries are updating their guidelines quite rapidly according to the dynamic new findings that surface every day. Continuous studies are necessary to know more and investigate further about the post-vaccine symptoms. To mitigate the above knowledge gap and support the national strategic preparedness and response plan, we undertook the following study which assesses the immediate symptoms associated with taking the Oxford-AstraZeneca COVID-19 vaccine among health care professionals.

\section{Methods}

\section{Study Area, Study Design, Sample Size, and Study Period}

This national online cross-sectional study was conducted from April 15-30, 2021 among health care providers aged greater than 18 years who took the first dose of OxfordAstraZeneca vaccine in Ethiopia. Sample size was determined by the online Raosoft sample size calculator (http:// www.raosoft.com/samplesize.html). In the first phase, the Ethiopian Ministry of Health intends to provide COVID19 vaccine to $20 \%$ of the population (about 23 million). We assumed a confidence level of $95 \%$, a margin of error of $3 \%$, an $80 \%$ response distribution, and the sample size recommended was 683 . For improving the quality of this study, we have used the Checklist for Reporting Results of Internet E-Survey (CHERRIES). ${ }^{18}$ In the present study, we have assessed the possible post-vaccine adverse effects, 
which might occur among participants who received the first dose of the vaccine.

\section{Development, Validation and Distribution of Survey Questionnaire}

This survey is conducted using 25 questions formulated in English language from different research publications, guidelines, and reports. ${ }^{1,12,14,19,20}$ The questions assessed the socio-demographic characteristics, presence of chronic diseases, injection site symptoms, mild symptoms, severe symptoms, previous COVID result, recommendation of the vaccine to others, and readiness to take the second dose. The constructed draft questionnaire was validated by seven selected healthcare professionals using the face and content validation approach to check its readability and validity prior to pretesting among fifteen randomly selected health professionals who took the COVID-19 AstraZeneca vaccine. In order to improve clarity, structure, and sequence of the questions, amendments were done according to the feedback received. The final survey link was distributed to health professionals via multiple media platforms such as Gmail, Facebook, Telegram, and WhatsApp in the form of a "Google Form" (see the Supplementary Material). Potential participants were directed to a page that featured a brief explanation to the study's objective, as well as directions on how to complete the survey. Prior to data collection, all respondents were received a standardized general invitation letter with the survey link to accept or decline to participate in the study, which included statements about voluntary participation and confidentiality. Participants who refused to the informed consent were not allowed to access the survey or participate in the study, and they were free to withdraw at any moment. We have collected the data as per the World Medical Association Declaration of Helsinki Ethical Principles. ${ }^{21}$ Individuals who clicked on the link were directed to Google forms, and in order to avoid missing data, participants were asked to complete all the survey questions or else they would be unable to move to the next step. Participants have received no incentives or rewards for their participation. ${ }^{22}$

\section{Statistical Analysis}

To ensure accuracy, all obtained data were entered into Microsoft Excel and cross-checked for the presence of any errors. The frequency table, graph, and mean with standard deviation are used to describe the data. Separate Logistic regression analysis was conducted to identify statistically significant factors that can be associated with different outcome variables (mild symptoms, severe symptoms, recommendation of the vaccine for others, and readiness to take the second dose). A p-value of less than 0.05 was considered to declare the statistically significance. Statistical analysis was performed using STATA version 14.1.

\section{Result}

In this study, there were 683 study participants that answered to our survey. After the data is cleaned, the analysis was conducted among 672 (98.4\%) participant with an average age of 29 years $( \pm 5.26)$. In the present study, $408(60.7 \%)$ of respondents were male and the majority of health care providers 627 (93.3\%) of the health care providers who took the vaccine had no stated comorbidity.

This study has assessed the various symptoms that could occur after the COVID-19 vaccination. Local effects on the injection site and some mild symptoms were the most common complaints reported by the study participants. Around $510(75.8 \%)$ of health care providers who took the vaccine reported injection site symptoms of pain (65.48\%) and tenderness $(57.89 \%)$. Approximately $60 \%$ of the study participants experienced the injection site symptom within 12 hours of vaccination, and the symptoms persisted roughly for about 24-72 hours among the majority $(63.53 \%)$ of the respondents. Among the health professionals who took part in this study, 105 (15.63\%) had Polymerase Chain Reaction (PCR) confirmed COVID-19 positive test, and out of this group, around 78 (74.29\%) received the vaccine six weeks after their positive test result. In our study, while $92 \%$ of the vaccinated respondents expressed their willingness to receive the second dosage, $94 \%$ recommended the vaccination to others (Table 1).

Among the study participants, 477 (70.98\%) reported mild symptoms where $350(52.08 \%)$ and 337 (50.1\%) have experienced tiredness and headache, respectively. Having difficulty related to sleep patterns and chills were the least reported mild symptoms with $20 \%$ and $27 \%$, respectively (Figure 1).

Only $54(11.32 \%)$ of respondents with mild symptoms were symptomatic 24 hours after being vaccinated. The duration when those mild symptoms stay ranges from one day to three days on most of the study participants $272(57.14 \%)$. According to the findings of this survey, 
Table I Characteristics of Health Professionals Who Took the First Dose of AstraZeneca Vaccine, Ethiopia, 202 I

\begin{tabular}{|c|c|c|c|}
\hline \multicolumn{2}{|l|}{ Variable } & \multirow{2}{*}{$\begin{array}{l}\text { Number } \\
264\end{array}$} & \multirow{2}{*}{$\begin{array}{l}\text { Percent } \\
39.3\end{array}$} \\
\hline Gender & Female & & \\
\hline & Male & 408 & 60.7 \\
\hline \multirow[t]{5}{*}{ Comorbidity } & Asthma & 15 & 2.23 \\
\hline & Hypertension & 14 & 2.08 \\
\hline & Diabetes & 7 & 1.04 \\
\hline & Other & 9 & 1.34 \\
\hline & No comorbidity & 627 & 93.3 \\
\hline \multirow[t]{2}{*}{ Presence of previously Confirmed COVID-19 case } & Yes & 105 & 15.63 \\
\hline & No & 567 & 84.38 \\
\hline \multirow[t]{2}{*}{ Time of vaccination after COVID-19 positive result $(n=105)$} & Within 6 weeks & 27 & 27.71 \\
\hline & $>6$ weeks & 78 & 74.29 \\
\hline \multirow[t]{2}{*}{ Recommending the vaccine for others } & Yes & 634 & 94.35 \\
\hline & No & 38 & 5.65 \\
\hline \multirow[t]{2}{*}{ Readiness to take the second dose of vaccine } & Yes & 619 & 92.11 \\
\hline & No & 53 & 7.89 \\
\hline \multirow[t]{2}{*}{ Injection site symptoms presence } & No & 162 & 24.2 \\
\hline & Yes & 510 & 75.8 \\
\hline \multirow[t]{4}{*}{ Types of Injection site symptoms $(n=5 \mid 0)$} & Pain & 440 & 65.48 \\
\hline & Tenderness & 389 & 57.89 \\
\hline & Swelling & 68 & 10.12 \\
\hline & Redness & 46 & 6.85 \\
\hline \multirow[t]{4}{*}{ Time to develop Injection site symptoms $(n=5 \mid 0)$} & Within 6 hours & 138 & 27.06 \\
\hline & $6-12$ hours & 171 & 33.53 \\
\hline & $12-24$ hours & 163 & 31.96 \\
\hline & After 24 hours & 38 & 7.45 \\
\hline \multirow[t]{4}{*}{ Duration of ISS $(n=5 \mid 0)$} & $<24$ hours & 63 & 12.35 \\
\hline & 24-72 hours & 324 & 63.53 \\
\hline & 72 hours-7 days & 113 & 22.16 \\
\hline & $>7$ days & 10 & 1.96 \\
\hline
\end{tabular}

most $287(60.29 \%)$ took pain killer to alleviate the mild symptoms they had (Table 2).

Forty-one $(6.1 \%)$ of health care professionals who had the vaccine's first shot reported severe symptoms marked by severe headache, shortness of breath, chest pain, leg swelling, rash, abdominal pain, severe muscle pain, and persistent cough. Among those who have experienced these severe symptoms, 26 (63.41\%) of participants felt the symptoms within 24 hours after vaccination, and majority $24(58.53 \%)$ of the severe symptoms lasted for 


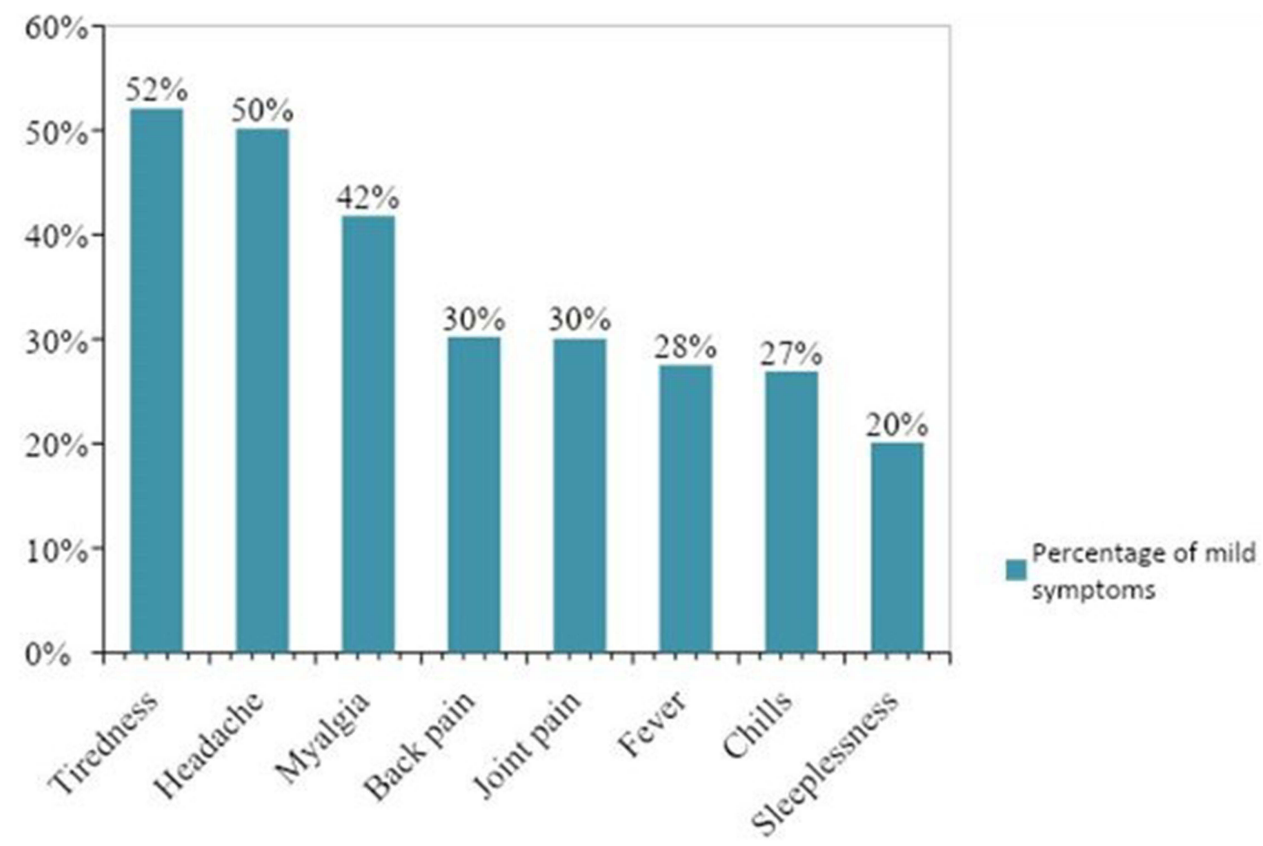

Figure I Percentage of mild symptoms reported by health care providers after taking the first dose of AstraZeneca COVID-19 vaccine, Ethiopia, 2021.

less than three days. Furthermore, none of the study participants who suffered adverse effects did seek medical help from health institutions. From those respondents who reported severe symptoms, none of them reported a laboratory-confirmed blood clotting problem (Table 3).

Among these health care providers, 38 (5.6\%) did not recommend the vaccine to others and $53(7.89 \%)$ had no plan of taking the second dose of the vaccine. The major $(39.5 \%)$ stated reasons for not recommending the vaccine to others were the fear of severe symptoms and other symptoms associated with blood clotting. The other reasons were the bad review they have heard about the vaccine $(31.6 \%)$, followed by the short time given for the vaccine development and doubt on the effectiveness of the vaccine $(15.8 \%)$; especially in preventing new strains of SARS-CoV-2. As for why they opted to not take the second dose, majority (56.6\%) anticipated that side effects may be severe with the second dose. There

Table 2 Characteristics of Mild Symptoms Reported Among Health Care Professionals Who Took the First Dose of AstraZeneca COVID-19 Vaccine, Ethiopia, 2021

\begin{tabular}{|c|c|c|c|}
\hline & & Frequency & Percent \\
\hline \multirow[t]{4}{*}{ Time to start mild symptoms } & $<6$ hours & 61 & 12.79 \\
\hline & $6-12$ hours & 174 & 36.48 \\
\hline & I2-24 hours & 188 & 39.41 \\
\hline & After 24 hours & 54 & 11.32 \\
\hline \multirow[t]{4}{*}{ Duration of mild symptoms } & $<24$ hours & 129 & 27.10 \\
\hline & $24-72$ hours & 272 & 57.14 \\
\hline & 72 hours -7 days & 57 & 11.97 \\
\hline & $>7$ days & 18 & 3.78 \\
\hline \multirow[t]{2}{*}{ Took pain killer for mild symptoms } & Yes & 287 & 60.29 \\
\hline & No & 189 & 39.71 \\
\hline
\end{tabular}


Table 3 Characteristics of Severe Symptoms Reported Among Health Care Professionals Who Took the First Dose of AstraZeneca COVID-19 Vaccine, Ethiopia, 2021

\begin{tabular}{|c|c|c|c|}
\hline & & Frequency & Percent \\
\hline \multirow[t]{8}{*}{ Severe symptoms } & Severe headache & 24 & 3.57 \\
\hline & Shortness of breath & 3 & 0.45 \\
\hline & Chest pain & 4 & 0.60 \\
\hline & Leg swelling & 2 & 0.30 \\
\hline & Rash & 5 & 0.74 \\
\hline & Abdominal pain & 6 & 0.89 \\
\hline & Severe muscle pain & 5 & 0.74 \\
\hline & Other & 12 & 1.79 \\
\hline \multirow[t]{3}{*}{ Time to start severe symptoms } & $<24$ hours & 26 & 63.41 \\
\hline & $24 \mathrm{hr}-7$ days & 13 & 31.71 \\
\hline & $>7$ days & 2 & 4.88 \\
\hline \multirow[t]{4}{*}{ Duration of severe symptoms } & $<24$ hours & 8 & $19.5 \mid$ \\
\hline & 24-72 hours & 16 & 39.02 \\
\hline & 72 hours -7 days & 7 & 17.07 \\
\hline & $>7$ days & 10 & 24.39 \\
\hline \multirow[t]{3}{*}{ Time to start severe symptoms } & $<24$ hours & 26 & 63.41 \\
\hline & $24 \mathrm{hr}-7$ days & 13 & 31.71 \\
\hline & $>7$ days & 2 & 4.88 \\
\hline \multirow[t]{4}{*}{ Duration of severe symptoms } & $<24$ hours & 8 & 19.51 \\
\hline & $24-72$ hours & 16 & 39.02 \\
\hline & 72 hours-7 days & 7 & 17.07 \\
\hline & $>7$ days & 10 & 24.39 \\
\hline \multirow{2}{*}{$\begin{array}{l}\text { Laboratory confirmed blood } \\
\text { clotting }\end{array}$} & Yes & 0 & 0.00 \\
\hline & No & 41 & 100.00 \\
\hline
\end{tabular}

were some individuals who have experienced some allergic reaction to the first dose of the vaccine and feared more severe allergic reaction to the second dose. Few of the study participants experienced vaccine-associated illnesses with severity worse than their experience of COVID-19 illness and hence thought they are better off without the second dose. Some study participants feared the long-term complications of the vaccine. Some others (3.8\%) had the understanding that a single dose of vaccine is adequate for those with previous COVID-19 infection and few (3.8\%) female participants of the study feared the uncertainty of the vaccine related with pregnancy.

We run different logistic regression analyses for mild symptoms, severe symptoms, recommendation of the vaccine for others, and readiness to take the second dose. The odds of having mild symptoms were 2.34 times higher among females than male (OR=2.34, 95\% CI $[1.62,3.30])$. There was no statistically significant variable associated with severe symptoms related to COVID-19 vaccine. The odds of recommending the vaccine to others were $83 \%$ less 
likely among those who developed severe symptoms than that of respondents with no severe symptoms $(\mathrm{OR}=0.17$, $95 \%$ CI [0.08, 0.39]). Similarly, the odds of willingness to take the second dose of the vaccine among respondents with severe symptoms were $85 \%$ less likely than those with no severe symptoms $(\mathrm{OR}=0.15,95 \% \mathrm{CI}[0.07,0.30])$.

\section{Discussion}

In this online-based study conducted in Ethiopia, we have investigated the adverse effects and determining factors following administration of the first dose of AstraZeneca COVID-19 vaccine among health care workers. A total of 672 health care workers participated in this study and reported the following symptoms during the first days of post vaccination. Among the symptoms, injection site symptoms (pain, tenderness, swelling, and redness) and mild symptoms (Tiredness, Headache, myalgia, back pain, joint pain, fever, chills, sleeplessness, nausea, sore throat, diarrhea, vomiting, runny nose, and cough) were commonly reported.

In this study, injection site symptoms were reported by $75.8 \%$ of healthcare workers who took the vaccine. These cutaneous reactions close to the injection site are common just as in other vaccines, ${ }^{5}$ and our result concurs well with a study conducted in Jordanian and Polish health workers. ${ }^{20,23}$ But this finding is higher than another prospective observational study conducted in the United Kingdom, which has shown injection site symptoms among $58.7 \%$ of study participants. ${ }^{19}$ Among the reported injection site symptoms, the commonest being injection site pain $(65.48 \%)$ followed by tenderness $(57.89 \%)$. This is relatively higher than other study where the injection site pain and tenderness were $54.2 \%$ and $63.7 \%$, respectively. ${ }^{24}$ Possible explanation for these discrepancies can be differences in study population (eg demographic data such as age), psychological differences in symptom reporting behavior, race-based pain threshold, ${ }^{25}$ sample size, and study design. ${ }^{26}$

This study provides us information on mild symptoms, which occurred among $70.98 \%$ of AstraZeneca first dose vaccine recipients. This result is lower than the Phase 2-3 trial of the ChAdOx1 nCoV-19 vaccine, which reported systemic adverse effects in $88 \%$ of participants aged 18 55 years who received the first injection. ${ }^{6}$

The finding of the mild symptom tiredness in our study is $52.08 \%$, which is consistent with the $53.1 \%$ finding from the other study. ${ }^{24}$ Additionally, the percentage of health care professionals who reported to have developed headache following the administration of the AstraZeneca COVID-19 vaccine is $50.15 \%$, which is in line with $52.6 \%$ from the other study. ${ }^{24}$ Around $42 \%$ of the study participants experienced myalgia with comparable findings, $44.0 \%$ in the other studies. ${ }^{20,24}$

Majority $(60.29 \%)$ of our respondents took pain killer to alleviate the mild symptoms that they encounter. Since identifying and managing adverse events following immunization (AEFI) is important to sustain trust in vaccines, WHO recommends taking analgesics for post-COVID-19 vaccine symptoms. However, CDC warns individuals not to take analgesics in a prophylactic manner for the interaction between the COVID-19 vaccine and an analgesic is not yet known. ${ }^{14}$

Most of the side effects $(84.24 \%)$ occurred within two or three days following vaccination, the majority of which are mild and local in nature, are expected and common. This was found to be consistent with other studies. ${ }^{27,28}$ However, from individuals who experience any severe symptoms $41(6.1 \%)$ - such as severe headache, chest pain, leg swelling, rash, abdominal pain, and severe muscle pain- those symptoms stayed for more than three days on $41.46 \%$ respondents. Furthermore, the WHO Global Advisory Committee recommended vaccine recipients to seek urgent medical care if the severe symptoms stays from four to twenty days after vaccination. ${ }^{27}$

\section{Conclusion and Recommendation}

As like that of other vaccines, the Oxford-AstraZeneca COVID-19 vaccine has some adverse effects and the most common symptoms, which can occur after vaccination were symptoms associated with the injection site and some mild symptoms. Most side effects peaked within the first 24 hours following vaccination and usually lasted 1-3 day. Severe symptoms were uncommon but found to be a significant reason for the vaccine recipients for not recommending it to others and also for not planning to take their second dose.

After the administration of the COVID-19 vaccine, recipients should be informed about possible vaccine symptoms, how to treat them, and when and from whom to seek additional advice if necessary. No significant association was found between previous positive result of COVID-19 test and any post-vaccine symptoms. We recommend further research to be conducted in the way of answering whether single dose of the COVID-19 vaccine is sufficient on those who had history of SARS-CoV-2 infection. 
Baseless speculations regarding the effects of vaccination can vary from partial-truths and unsubstantiated assumptions to deliberate misinformation based on conspiracy theories. Misinformation, no matter how distant from our present scientific understanding, it may affect people's views and decision-making if left unchecked, contributing to a self-perpetuating cycle of negative news. Therefore, all responsible stakeholders should battle misinformation by aggressively disseminating factual information regarding risks and benefits of the vaccination.

\section{Limitation of the Study}

First, although we attempted to employ a representative sample of health care professionals, we cannot be certain how representative survey respondents are of the general health care professionals. Second, some variables such as time to develop the symptoms after vaccinated and the duration that the symptoms stayed might be prone to recall bias. Third, because this is an internet-based online survey, respondents from locations without internet access may be missed, resulting in demographic selection bias.

\section{Data Sharing Statement}

Supporting data for the current study are available from the corresponding author on reasonable request.

\section{Ethical Approval and Informed Consent}

This study has been approved by the Research Ethical review Committee of School of Medicine, Debre Markos University (S/R/C/47/03/13). Prior to the main data collection, informed consent was acquired after the studies were well explained.

\section{Acknowledgment}

We would like to thank the study participants and those who genuinely share the online survey link.

\section{Author Contributions}

All authors made a significant contribution to the work reported, whether that is in the conception, study design, execution, acquisition of data, analysis and interpretation, or in all these areas; took part in drafting, revising or critically reviewing the article; gave final approval of the version to be published; have agreed on the journal to which the article has been submitted; and agree to be accountable for all aspects of the work.

\section{Funding}

The authors received no funding from an external source.

\section{Disclosure}

The authors declare no conflicts of interest for this work.

\section{References}

1. World Health Organization. WHO coronavirus disease (COVID-19) dashboard. 2021; Available from: https://covid19.who.int/. Accessed September 10, 2021.

2. UNCTAD. Impact of the COVID-19 pandemic on trade and development. March 31, 2021; Available from: https://unctad.org/ programme/covid-19-response/impact-on-trade-and-development -2021. Accessed September 10, 2021.

3. World Health Organization. COVID-19 strategic preparedness and response plan: operational planning guideline: 1 February 2021 to 31 January 2022. World Health Organization; 2021.

4. Khuroo MS, Khuroo M, Khuroo MS, et al. COVID-19 vaccines: a race against time in the middle of death and devastation! J Clin Exp Hepatol. 2020;10(6):610-621. doi:10.1016/j.jceh.2020.06.003

5. Bogdano G, Bogdanov I, Kazandjieva J, et al. Cutaneous adverse effects of the available COVID-19 vaccines. Clin Dermatol. 2021. doi:10.1016/j.clindermatol.2021.04.001

6. Ramasamy MN, Minassian AM, Ewer KJ, et al. Safety and immunogenicity of ChAdOx1 nCoV-19 vaccine administered in a prime-boost regimen in young and old adults (COV002): a single-blind, randomised, controlled, phase $2 / 3$ trial. Lancet. 2020;396(10267):1979-1993. doi:10.1016/S0140-6736(20)32466-1

7. World Health Organization. Ethiopia introduces COVID-19 vaccine in a national launching ceremony. 2021.

8. Solís Arce JS, Warren SS, Meriggi NF, et al. COVID-19 vaccine acceptance and hesitancy in low- and middle-income countries. Nat Med. 2021;27(8):1385-1394. doi:10.1038/s41591-021-01454-y

9. Saied SM, Saied EM, Kabbash IA, et al. Vaccine hesitancy: beliefs and barriers associated with COVID-19 vaccination among Egyptian medical students. J Med Virol. 2021;93(7):4280-4291. doi:10.1002/ jmv. 26910

10. Machingaidze S, Wiysonge CS. Understanding COVID-19 vaccine hesitancy. Nat Med. 2021;27(8):1338-1339. doi:10.1038/s41591021-01459-7

11. Rief W. Fear of adverse effects and COVID-19 vaccine hesitancy: recommendations of the treatment expectation expert group. JAMA health forum. American Medical Association; 2021.

12. Jayadevan R, Shenoy RS, Anithadevi T. Survey of symptoms following COVID-19 vaccination in India. medRxiv. 2021. doi:10.1101/ 2021.02.08.21251366.

13. Wise J. Covid-19: How AstraZeneca lost the vaccine PR war. BMJ. 2021;373:n921.

14. Centers for Disease Control and Prevention. Possible side effects after getting a COVID-19 vaccine; May 25, 2021 [cited May 25, 2021]. Available from: https://www.cdc.gov/coronavirus/2019-ncov /vaccines/expect/after.html. Accessed September 10, 2021.

15. Andrzejczak-Grządko S, Czudy Z, Donderska M. Side effects after COVID-19 vaccinations among residents of Poland. Eur Rev Med Pharmacol Sci. 2021;25(12):4418-4421.

16. Klugar M, Riad A, Mekhemar M, et al. Side effects of mRNA-based and viral vector-based COVID-19 vaccines among German healthcare workers. Biology. 2021;10(8):752. doi:10.3390/biology10080752

17. Riad A, Pokorná A, Mekhemar M, et al. Safety of ChAdOx1 nCoV-19 vaccine: independent evidence from two EU states. Vaccines. 2021;9(6):673. doi:10.3390/vaccines 9060673 
18. Eysenbach G. Improving the quality of web surveys: the checklist for reporting results of internet E-surveys (CHERRIES). $J$ Med Internet Res. 2004;6(3):e34. doi:10.2196/jmir.6.3.e34

19. Menni C, Klaser K, May A, et al. Vaccine side-effects and SARS-CoV-2 infection after vaccination in users of the COVID symptom study app in the UK: a prospective observational study. Lancet Infect Dis. 2021;21 (7):939-949. doi:10.1016/S1473-3099(21)00224-3

20. Abu-Hammad O, Alduraidi H, Abu-Hammad S, et al. Side effects reported by Jordanian healthcare workers who received COVID-19 vaccines. Vaccines. 2021;9(6):577. doi:10.3390/vaccines9060577

21. General Assembly of the World Medical Association. World Medical Association Declaration of Helsinki: ethical principles for medical research involving human subjects. $J$ Am Coll Dent. 2014;81(3):14-18.

22. Von Elm E, Altman DG, Egger M, et al. The Strengthening the Reporting of Observational Studies in Epidemiology (STROBE) statement: guidelines for reporting observational studies. Bull World Health Organ. 2007;85(11):867-872. doi:10.2471/BLT.07.045120

23. Jęśkowiak I, Wiatrak B, Grosman-Dziewiszek P, et al. The incidence and severity of post-vaccination reactions after vaccination against COVID-19. Vaccines. 2021;9(5):502. doi:10.3390/vaccines9050502
24. Voysey M, Clemens SA, Madhi SA, et al. Safety and efficacy of the ChAdOx1 nCoV-19 vaccine (AZD1222) against SARS-CoV-2: an interim analysis of four randomised controlled trials in Brazil, South Africa, and the UK. Lancet. 2021;397(10269):99-111.

25. Hervé C, Laupèze B, Del Giudice G, et al. The how's and what's of vaccine reactogenicity. npj Vaccines. 2019;4(1):1-11. doi:10.1038/ s41541-019-0132-6

26. McCambridge J, Kypri K, Elbourne D. Research participation effects: a skeleton in the methodological cupboard. J Clin Epidemiol. 2014;67(8):845-849. doi:10.1016/j.jclinepi.2014.03.002

27. World Health Organization. Interim statement of the COVID-19 subcommittee of the WHO Global Advisory Committee on vaccine safety on AstraZeneca COVID-19 vaccine. Saudi Med J. 2021;42(5):581-582.

28. Alhazmi A, Alamer E, Daws D, et al. Evaluation of side effects associated with COVID-19 vaccines in Saudi Arabia. Vaccines. 2021;9(6):674. doi:10.3390/vaccines9060674
Journal of Multidisciplinary Healthcare

\section{Publish your work in this journal}

The Journal of Multidisciplinary Healthcare is an international, peerreviewed open-access journal that aims to represent and publish research in healthcare areas delivered by practitioners of different disciplines. This includes studies and reviews conducted by multidisciplinary teams as well as research which evaluates the results or conduct of such teams or healthcare processes in general. The journal

\section{Dovepress}

covers a very wide range of areas and welcomes submissions from practitioners at all levels, from all over the world. The manuscript management system is completely online and includes a very quick and fair peer-review system. Visit http://www.dovepress.com/testimonials. php to read real quotes from published authors. 